\title{
Universal Quantum Computation with Continuous-Variable Abelian Anyons
}

\author{
Darran F. Milne ${ }^{1}$, Natalia V. Korolkova ${ }^{1}$, Peter van Loock $^{2}$ \\ ${ }^{1}$ School of Physics and Astronomy, University of St. Andrews, \\ North Haugh, St. Andrews, Fife, KY16 9SS, Scotland, \\ ${ }^{2}$ Optical Quantum Information Theory Group, Max-Planck Institute for the Science of Light, \\ Günther-Scharowsky-Str.1/Bau 26 and Institute of Theoretical Physics, \\ Universität Erlangen-Nürnberg, Staudtstr. 7/B2, 91058 Erlangen, Germany
}

\begin{abstract}
We describe how continuous-variable abelian anyons, created on the surface of a continuousvariable analogue of Kitaev's lattice model can be utilized for quantum computation. In particular, we derive protocols for the implementation of quantum gates using topological operations. We find that the topological operations alone are insufficient for universal quantum computation which leads us to study additional non-topological operations such as offline squeezing and single-mode measurements. It is shown that these in conjunction with a non-Gaussian element allow for universal quantum computation using continuous-variable abelian anyons.
\end{abstract}

\section{INTRODUCTION}

The efficient storage and processing of quantum information is a major open problem for quantum computation (QC). One of the main routes to protect quantum information against random errors introduced via environmental decoherence and noise is the use of quantum error correcting (QEC) codes [1-4]. Essentially, such schemes attempt to correct errors with clever 'software' design. In contrast, recently, a new approach has been suggested in which information is intrinsically protected by storing it as a non-local topological degree of freedom. This approach is known as Topological Quantum Computation (TQC). In these schemes, ideally, the protection is an intrinsic property of the physical system, being inaccessible to noise. Moreover, any quantum gates that are implemented by non-trivial topological operations are also protected.

A recent development in TQC is the realisation of certain exotic states of matter that occur in two dimensions, the so-called anyon states, which use these topological properties of a system to provide a natural medium for storing and manipulating quantum information $[5,6]$. Anyons are quasiparticle excitations that exhibit fractional statistics, i.e., when two anyons are exchanged the quantum state acquires a phase shift corresponding to the fractional spin of the anyonic states. These anyonic states first emerged in connection with the fractional quantum Hall effect [7], which occurs in a two-dimensional electron gas at low temperatures. Anyons have been extensively studied for their fundamental interest, but recently it has been shown that anyons are a useful resource for fault-tolerant topological quantum computation [8-10]. Anyons fall into two general classes; abelian and nonabelian. Non-abelian anyons have received the most attention as it has been shown that some species, notably the Fibonacci anyons [9], are a resource for universal QC over discrete variables using just their braiding and fusion operations alone. However, it has been shown that the operations available to abelian anyons can also provide a universal gate set [11-13], but certain operations such as single-qubit rotations, must be carried out using non-topological methods.

A simple model from which one can produce abelian anyons was proposed by Kitaev for spin $1 / 2$ systems $[8,14]$. However, this surface code model involves suitable combinations of four-body interactions which are difficult to achieve experimentally. In [15] it was shown that this code can be created efficiently from a two-dimensional cluster state by selectively measuring out single spins. This protocol was extended to the Continuous-Variable (CV) regime in [16] where it was shown that from a CV cluster state, a CV analogue of the Kitaev model can be constructed. It was demonstrated that the continuous excitations above this ground state are $\mathrm{CV}$ abelian anyons with non-trivial braiding statistics. Here we study the computational power of these $\mathrm{CV}$ anyons. We show how to create gates based on their topological properties and find that we can implement single-mode phase-space displacements as well as twomode controlled phase-space displacements, where both the control and the target can be either in the computational or in the conjugate basis. However, analogous to the qubit case, we find that the topological operations for the CV abelian anyons do not form a sufficient gate set for universal QC and so we include certain non-topological operations to supplement the topological operations in order to complete the gate set.

We begin, in Sec. II, with a short introduction to quantum computation over continuous variables. In Sec. III, we review briefly how to construct the CV Kitaev ground state from a CV cluster state and discuss the fusion and braiding properties of the anyonic excitations. In Sec. IV we examine the range of Clifford group operations that are achievable with the $\mathrm{CV}$ anyons by topological and non-topological actions. The Clifford gates alone are not enough for universal CV QC and hence in Sec. V, we study the effect on the anyons of applying a cubic phase gate to the Kitaev ground state. In Secs. VI and VII, we investigate the effect of finite squeezing of the resource state on the excitations and computational model. We conclude in Sec. VIII. 


\section{CONTINUOUS-VARIABLE QUANTUM COMPUTATION}

Quantum logic over continuous variables generalizes the qubit Pauli $X$ and $Z$ operators to the WeylHeisenberg (WH) group [17], the group of phase space displacements. This is a Lie group with generators $\hat{x}=\left(\hat{a}+\hat{a}^{\dagger}\right) / \sqrt{2}$ and $\hat{p}=i\left(\hat{a}^{\dagger}-\hat{a}\right) / \sqrt{2}$ representing, for instance, a single quantized mode (qumode) of the electromagnetic field. These operators satisfy the canonical commutation relation $[\hat{x}, \hat{p}]=i$, equivalent to position and momentum. In what follows, we refer to $\hat{x}$ and $\hat{p}$ as position and momentum. The single-mode WH operators are defined as $X(s)=e^{-i s \hat{p}}$ and $Z(t)=e^{i t \hat{x}}, s, t \in \mathbb{R}$. The WH operator $X(s)$ is the position-translation operator, which acts on the computational basis of position eigenstates $\{|x\rangle ; x \in \mathbb{R}\}$ as $X(s)|x\rangle=|x+s\rangle . Z(t)$ is the momentum-translation operator, which acts on the momentum eigenstates $\{|p\rangle ; p \in \mathbb{R}\}$ as $Z(t)|p\rangle=|p+t\rangle$. These operators are non-commutative and obey the identity

$$
X(s) Z(t)=e^{-i s t} Z(t) X(s) .
$$

In the following we will show how a CV computational model can be constructed using $\mathrm{CV}$ abelian anyons.

\section{GENERATION OF ANYONIC STATES FROM CV GRAPH STATES}

We review the protocol of [16], for the generation of the CV Kitaev ground state. This scheme is based on the first Kitaev lattice model which is a spin Hamiltonian for a two-dimensional square lattice. In Kitaev's scheme, a qubit (for instance, a spin $1 / 2$ particle) is associated with each edge of a square lattice, for which the model Hamiltonian is given by

$$
H=-\sum_{s} A_{s}-\sum_{f} B_{f}
$$

where $A_{s}=\prod_{j \in \operatorname{star}(s)} X_{j}$, and $B_{f}=\prod_{j \in \partial(f)} Z_{j}$. Here $\partial(f)$ denotes the boundary spins of a plaquette and the operators $X$ and $Z$ are the standard Pauli matrix operators $\sigma_{x}$ and $\sigma_{z}$.

These four-body interactions can be produced experimentally by cooling the system to its ground state, but a more practical approach suggested in [15] is to create the ground state dynamically from graph or cluster states. In [16] this model was generalized to CV states which we turn to now.

In the ideal case, CV cluster states are prepared from a collection of $N$ zero-momentum eigenstates [18-21], which we write as $|0\rangle_{p}^{\otimes N}$, where the $p$-subscripted kets satisfy $\hat{p}|s\rangle_{p}=s|s\rangle_{p}$. These states are then entangled via a collection of controlled-Z operations, denoted $C_{Z}=\exp \left(i g \hat{x}_{i} \otimes \hat{x}_{j}\right)$, where $g \in \mathbb{R}$ is the strength of

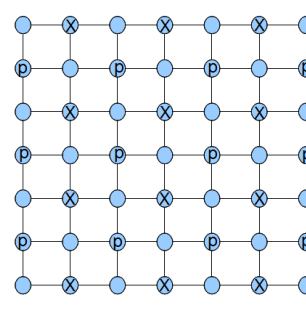

(a)

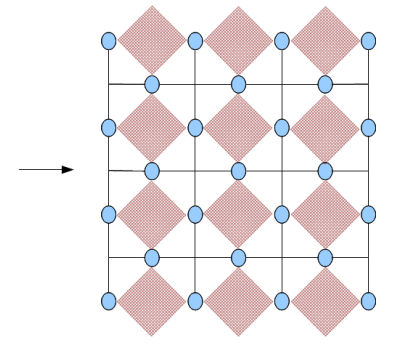

(b)
FIG. 1: (a) The measurement pattern to prepare the anyonic ground state from a two-dimensional cluster state, where $X$ and $P$ denote a single-mode measurement in the position and momentum bases, respectively. (b) The CV surface code model.

the interactions (we will assume $g=1$ throughout). Labelling the nodes of the graph in some arbitrary order, we can define a symmetric adjacency matrix $\mathbf{A}=\mathbf{A}^{T}$ whose $(j, k)$ th entry $A_{j k}$ is equal to the weight of the edge linking node $j$ to node $k$ (with no edge corresponding to a weight 0 ). Note in the ideal case the diagonal entries are all zero since we do not allow for self loops in the graph [22]. The collection of controlled- $Z$ operations used to make the CV cluster state is then a function of $\mathbf{A}$, denoted $C_{Z}[\mathbf{A}]$. The $\mathrm{CV}$ cluster state with the graph A is then

$$
\begin{aligned}
\left|\psi_{A}\right\rangle & =C_{Z}[\mathbf{A}]|0\rangle_{p}^{\otimes N} \\
& =\prod_{j, k=1}^{N} \exp \left(\frac{i}{2} A_{j k} \hat{x}_{j} \hat{x}_{k}\right)|0\rangle_{p}^{\otimes N} \\
& =\exp \left(\frac{i}{2} \hat{\mathbf{x}}^{T} \mathbf{A} \hat{\mathbf{x}}\right)|0\rangle_{p}^{\otimes N}
\end{aligned}
$$

where $\hat{\mathbf{x}}=\left(\hat{x}_{1}, \ldots, \hat{x}_{N}\right)^{T}$ is a column vector of position operators. Ideal CV cluster states in the unphysical limit of infinite squeezing satisfy a set of nullifier relations, which can be written as

$$
(\hat{\mathbf{p}}-\mathbf{A} \hat{\mathbf{x}})\left|\psi_{A}\right\rangle=0,
$$

where $\hat{\mathbf{p}}=\left(\hat{p}_{1}, \ldots, \hat{p}_{N}\right)^{T}$ is a column vector of momentum operators. This represents $N$ independent equations, one for each component of the vector $(\hat{\mathbf{p}}-\mathbf{A} \hat{\mathbf{x}})$, which are the nullifiers for $\left|\psi_{A}\right\rangle$, because that state is a simultaneous zero-eigenstate of them. The nullifiers are written explicitly as

$$
\hat{g}_{a}=\left(\hat{p}_{a}-\sum_{b \in N_{a}} \hat{x}_{b}\right) \rightarrow 0, \quad \forall a \in G,
$$

where the modes $a \in G$ correspond to the vertices of the graph of $N$ modes and the modes $b \in N_{a}$ are the nearest neighbours of mode $a$. An efficient way of representing CV cluster states is via the stabilizer formalism. A state 
$|\phi\rangle$ is stabilized by an operator $K$ if it is an eigenstate of $K$ with unit eigenvalue. If such a set exists for a given state, then we call the state a stabilizer state, and we may use the generators of its stabilizer group to uniquely specify it. The stabilizer operator for a cluster state is $G_{a}(\xi)=\exp \left(-i \xi \hat{g}_{a}\right)=X_{a}(\xi) \prod_{b \in N_{a}} Z_{b}(\xi)$.

To construct the CV Kitaev code state from a CV cluster state, we make single-mode measurements on every second mode in either the position or momentum basis, with a subsequent Fourier transform, $F$, for all the remaining modes which leaves us with a new graph state $|\psi\rangle$ (Fig. 1). This new state is described by the set of correlations of the form $\left\{\hat{a}_{s}=\left(\hat{p}_{s, 1}+\hat{p}_{s, 2}+\hat{p}_{s, 3}+\right.\right.$ $\left.\left.\hat{p}_{s, 4}\right) \rightarrow 0, \quad \hat{b}_{f}=\left(\hat{x}_{f, 1}-\hat{x}_{f, 2}+\hat{x}_{f, 3}-\hat{x}_{f, 4}\right) \rightarrow 0\right\}$ where $s$ and $f$ label star and plaquettes, respectively, and the indices $1, . ., 4$ of the position and momentum operators denote those modes located at a common star or at the boundary of a common plaquette. The new stabilizer operators that describe this state, $\left\{A_{s}(\xi)=\right.$ $\exp \left(-i \xi \hat{a}_{s}\right)=\prod_{j \in \operatorname{star}(s)} X_{s, j}(\xi), B_{f}(\eta)=\exp \left(-i \eta \hat{b}_{f}\right)=$ $\left.\prod_{j \in \partial(f)} Z_{f, j}\left((-1)^{j} \eta\right)\right\}$ with $\xi, \eta \in \mathbb{R}$, are analogous to the first Kitaev model for a two-dimensional spin lattice. Since these new stabilizer operators commute, the new ground state corresponds to an anyonic ground state with $A_{s}(\xi)|\psi\rangle=|\psi\rangle$ and $B_{f}(\xi)|\psi\rangle=|\psi\rangle$ for all stars $s$ and plaquettes $f$, in the limit of infinite squeezing.

We regard continuous excitations above the preprepared ground state $|\psi\rangle$ as CV anyons, produced by applying $Z$ and $X$ operators on the ground state. Specifically, the position-translation operator applied to some mode of the lattice creates a pair of $m$-type anyons on adjacent plaquettes, i.e., $\left|m\left((-1)^{d} t\right)\right\rangle=X(t)|\psi\rangle(d \in$ $\{1,2\})$, where $d=1$ means the relevant mode lies on the vertical edges, and $d=2$ refers to the horizontal edges). An e-type pair of anyons is created on adjacent vertices of the lattice by $|e(s)\rangle=Z(s)|\psi\rangle$. We define a computational basis $|r\rangle_{v / f}$ on vertices, $v$, or plaquettes, $f$, with $r \in \mathbb{R}$, composed of a pair of anyons as

$$
\begin{aligned}
|r\rangle_{v} & =|e(r)\rangle_{v_{1}}|e(-r)\rangle_{v_{2}}, \\
|r\rangle_{f} & =|m(r)\rangle_{f_{1}}|m(-r)\rangle_{f_{2}} .
\end{aligned}
$$

The fusion rules $e(s) \times e(t)=e(s+t), \quad m(s) \times m(t)=$ $m(s+t), \quad e(0) \times e(s)=e(s), \quad m(0) \times m(s)=m(s)$ describe the outcome of combining two anyons. By application of a sequence of $X$ and $Z$ operators we can braid the anyons. For example, consider an initial state $\left|\psi_{\text {ini }}\right\rangle=Z_{i}(s)|\psi\rangle=|e(s)\rangle$. If an anyon of type $m$ is at a neighbouring plaquette, it can be moved around $e$ along a path generated by successive application of $X(t)$ on the four modes of the star. The final state is

$$
\begin{aligned}
\left|\psi_{\text {fin }}\right\rangle & =X_{1}(t) X_{2}(t) X_{3}(t) X_{4}(t)\left|\psi_{\text {ini }}\right\rangle \\
& =e^{-i s t} Z_{i}(s)\left[X_{1}(t) X_{2}(t) X_{3}(t) X_{4}(t)|\psi\rangle\right] \\
& =e^{-i s t}\left|\psi_{\text {ini }}\right\rangle .
\end{aligned}
$$

The phase factor is known as the topological phase factor, which reveals the presence of enclosed anyons. Note that the same phase factor would be acquired by the state independent of the path the braiding anyon follows. This topological character reveals the potential robustness of operations with CV anyons and their use as a resource for fault-tolerant quantum computation.

\section{CLIFFORD GATES}

We now examine the computational power of the $\mathrm{CV}$ abelian anyons. In particular we show how the Clifford group operations are achieved using both topological and non-topological means. The set $\left\{Z(s), F, P(\eta), C_{Z} ; s, \eta \in\right.$ $\mathbb{R}\}$ generates the Clifford group [17], where $P(\eta)=$ $\exp \left[i(\eta / 2) \hat{x}^{2}\right]$ is a CV squeezing gate, $F=\exp \left[i \pi / 4\left(\hat{x}^{2}+\right.\right.$ $\left.\left.\hat{p}^{2}\right)\right]$ is the Fourier Transform operator, and $C_{Z}=$ $\exp \left(i g \hat{x}_{i} \otimes \hat{x}_{j}\right)$ is the controlled-Z gate, as defined before. Transformations within the Clifford group correspond to Gaussian transformations mapping Gaussian states onto Gaussian states. We see below that the topological operations available to CV abelian anyons are not sufficient to generate the entire group and we will require nontopological operations to complete the set.

\section{A. Topological Operations}

Our first topological operations are quadrature displacements, $Z(s)=e^{i s \hat{x}}$. Phase-space displacements are achieved through the creation and fusion of anyons. It is easy to see that creation of an anyon results in a displacement away from the ground state. For non-trivial displacements we fuse anyons of the same type, created by displacements on modes $i$ and $j$. The anyon on site $j$ can then be moved to site $i$ to implement fusion,

$$
\begin{aligned}
|e(s)\rangle \times|e(t)\rangle & =Z_{i}(s)|\psi\rangle \times Z_{j}(t)|\psi\rangle \\
& =[Z(s) \times Z(t)]_{i}|\psi\rangle \\
& =e^{i(s+t) \hat{x_{i}}}|\psi\rangle .
\end{aligned}
$$

To act our quadrature displacement on the computational basis we must ensure that both anyons in the produced pair are fused with its counterpart. Hence the effect of our displacement on the computational basis is

$$
|r+s\rangle_{v}=|e(r+s)\rangle_{v_{1}}|e(-(r+s))\rangle_{v_{2}} .
$$

The change in the computational basis for the $m$-type anyons follows similarly. We can extend this to the two mode SUM gate (Fig. 2), which is a controlled displacement $C_{X}=e^{-i \hat{x}_{i} \otimes \hat{p}_{j}}$, i.e., $|x\rangle_{1}|y\rangle_{2} \rightarrow|x\rangle_{1}|x+y\rangle_{2}$. We affect the SUM gate by fusing one of the anyons from the first mode with an anyon from the second mode. This results in a displacement of the second mode dependant on the state of the first:

$$
\begin{aligned}
|s\rangle_{1}|t\rangle_{2} & =(|e(s)\rangle|e(-s)\rangle)_{1}(|e(-t)\rangle|e(t)\rangle)_{2} \\
& \rightarrow|e(-s)\rangle_{1}|e(s+t)\rangle_{2} \\
& =|-s\rangle_{1}|s+t\rangle_{2}
\end{aligned}
$$


where we treat the second anyon of mode two as a spectator anyon that will be annihilated at the end of the computation and the control is left in the original state (up to a sign change).

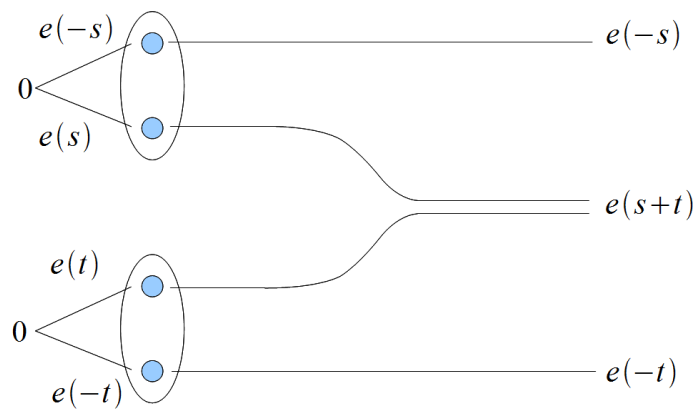

FIG. 2: The two-mode SUM gate. An anyon from the top pair is fused to produce a controlled displacement on the lower pair.

We can also perform a controlled-Z gate (Fig. 3), which acts on the computational basis as $|x\rangle_{1}|y\rangle_{2} \rightarrow|x\rangle_{1} e^{i \phi}|y\rangle_{2}$. This time we affect this transformation by braiding the anyons. For example to perform a controlled displacement in the conjugate basis on an $m$-type anyon we braid an $e$-type anyon around it. From equation (7), we see that the state picks up a phase dependant on the anyonic states.

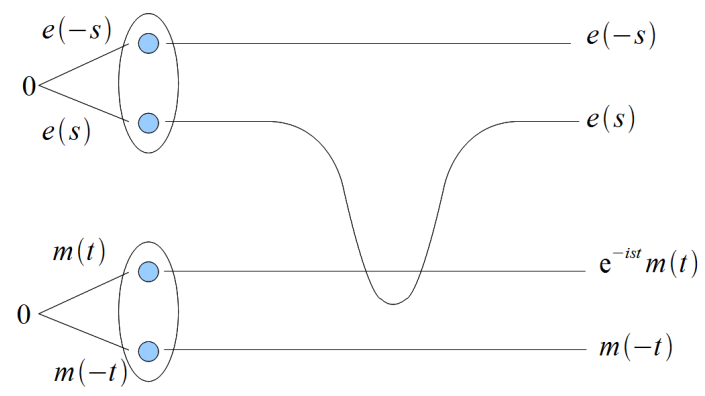

FIG. 3: A controlled phase gate $C_{Z}$. Braiding an anyon from the top pair with the bottom pair produces a phase change dependant on the state of the state of the anyons.

Hence, we have found that the controlled shift operation $C_{X}, e^{i \hat{x}_{i} \hat{p}_{j}}$, corresponds to partial fusions of $e$ type anyon pairs, while the controlled shift operation $C_{Z}$, $e^{i \hat{x}_{i} \hat{x}_{j}}$, corresponds to partial braiding between $e$ - and $m$ type anyon pairs. Similarly, we note that the operations $e^{i \hat{p}_{i} \hat{p}_{j}}$ and $e^{i \hat{p}_{i} \hat{x}_{j}}$ are achieved by partial braiding between $m$ and $e$-type anyon pairs and partial fusion of $m$-anyon pairs, respectively.

\section{B. Non-Topological Operations}

Above we saw how we can implement single mode displacements, displacements in the conjugate basis and two-mode controlled displacement gates. Now we go beyond simple first-moment Gaussian operations and consider the manipulation of second moments. In particular, we seek to complete the set of Clifford gates by the inclusion of a squeezer and a Fourier transform. The squeezing operation compresses the position quadrature by a factor $\eta$ while stretching the conjugate quadrature by $1 / \eta$. We cannot directly squeeze our anyons since the only action we can take on the anyons is fusion and braiding. Instead, we squeeze on some mode $i$ of the ground state,

$$
|\tilde{\psi}\rangle=P_{i}(\eta)|\psi\rangle
$$

Then creating an anyon on this squeezed ground state mode and commuting through gives us

$$
\begin{aligned}
Z_{i}(s)|\tilde{\psi}\rangle & =Z_{i}(s) P_{i}(\eta)|\psi\rangle \\
& =P_{i}(\eta) e^{i 2 \eta s \hat{x}_{i}} Z_{i}(s)|\psi\rangle
\end{aligned}
$$

We find that squeezing the ground state is equivalent to squeezing the anyon with an additional phase space displacement. This squeezing operation combined with a measurement in the $X$ basis can be used to implement a Fourier transform $F$. The action of $F$ is to switch between the position and momentum bases, i.e., $F|x\rangle_{x}=|x\rangle_{p}$. This corresponds to a generalization of the Hadamard gate for qubits. To perform $F$ on our anyons, we begin by preparing a zero-momentum squeezed ground state $|0\rangle_{p}$. We proved above that up to a displacement, any squeezer on the ground state acts on the anyonic excitations in the same way. Hence we can produce zero-momentum anyons. For example, consider an $m$-type anyon in the computational basis $\left|\psi^{\prime}\right\rangle=X(s) \int d t f(t)|t\rangle_{q}$. We fuse this anyon with the momentum squeezed anyon:

$$
\begin{aligned}
\operatorname{SUM}\left[\left|\psi^{\prime}\right\rangle \times|0\rangle_{p}\right] & =\operatorname{SUM}\left[X(s)|\psi\rangle \times|0\rangle_{p}\right] \\
& =\operatorname{SUM}\left[\int d t f(t) X(s)|t\rangle_{q}|0\rangle_{p}\right] \\
& =\int d t f(t)|t+s\rangle_{q}|t+s\rangle_{p} .
\end{aligned}
$$

Then, performing a measurement of $\hat{p}$ with outcome $m$ on the mode corresponding to the first anyon collapses this to

$$
\int d t f(t) e^{i(t+s) m}|s+t\rangle_{p}=X(m) F\left|\psi^{\prime}\right\rangle .
$$

We see that the effect of this procedure is to apply a Fourier transform modulo a known quadrature displacement. This completes our set of Clifford gates, and so with appropriate non-topological operations we can apply any Gaussian transformation. As stated above, Gaussian transformations are not sufficient for universal QC and we address the question of universality in the next section. 


\section{NON-CLIFFORD GATES AND UNIVERSALITY}

For our abelian anyon computational scheme, we require either a non-Gaussian gate or a non-Gaussian resource. In a similar fashion to our squeezing operation, we apply a transformation known as a cubic phase [23], $V(\gamma)=e^{i \gamma \hat{x}^{3}}, \gamma \in \mathbb{R}$, to some mode $i$ of the ground state. Then we commute $V(\gamma)$ through the $X$ and $Z$ operators to find the effect on the anyons:

$$
\left|\psi_{c}\right\rangle=V_{i}(\gamma)|\psi\rangle
$$

Now applying $X$ and $Z$ operators to create $e$ - and $m$-type anyons and commuting the cubic phase gate through we find

$$
\begin{aligned}
X_{i}(s) Z_{i}(t)\left|\psi_{c}\right\rangle & =X_{i}(s) Z_{i}(t) V_{i}(\gamma)|\psi\rangle \\
& =V_{i}(\gamma) e^{-i\left(s \hat{p}_{i}-t \hat{x}_{i}+3 \gamma s \hat{x}_{i}^{2}\right)} X_{i}(s) Z_{i}(t)|\psi\rangle
\end{aligned}
$$

Hence we find that applying a cubic phase to the ground state is equivalent to applying the cubic phase to the anyons with extra displacements and squeezing operations. This produces non-Gaussian anyons that can then be used to obtain a universal gate set. To conclude our discussion, we attempt to provide a classification of our gate operations. We found that robust topological operations correspond to controlled or uncontrolled $\mathrm{WH}$ gates. Non-robust, non-topological Clifford operations correspond to symplectic operations. Non-robust, nontopological, non-Clifford operations correspond to nonWH, non-symplectic operations. In group theoretical terms, all operations that are not elements of the normalizer of the WH group (Clifford group) cannot be topologically realized using abelian anyons. Of those operations that are elements of the normalizer of $\mathrm{WH}$, only the elements of the normal subgroup of the Clifford group can be topologically realized using abelian anyons; those Clifford elements that are not elements of the normal subgroup cannot be realized topologically using abelian anyons [24].

\section{PHYSICAL STATES - FINITE SQUEEZING}

So far, we have only considered the generation of anyonic statistics on an infinitely squeezed ground state. However, this state is highly unphysical. Here we extend our model to include finite squeezing of the initial state and show what effects this has on our computational model.

A method to extend the graph representations from ideal (infinitely squeezed) CV cluster states to their finitely squeezed Gaussian approximations was given in [22]. There it was shown that the nullifier formalism for $\mathrm{CV}$ cluster states can be extended to general Gaussian pure states using the simple replacement of the CV cluster state graph $\mathbf{A}$ with the Gaussian graph $\mathbf{Z}$, so that
$(\hat{\mathbf{p}}-\mathbf{Z} \hat{\mathbf{q}})\left|\phi_{Z}\right\rangle=0$ with the new non-Hermitian nullifiers defined as

$$
g_{k}=\hat{p}_{k}-i e^{-2 r_{k}} \hat{x}_{k}-\sum_{l \in N_{k}} \hat{x}_{l}, \quad \forall k .
$$

Then the adjacency matrix $\mathbf{Z}$ for a Gaussian pure state is a complex matrix with imaginary diagonal entries, $i e^{-2 r_{k}}$, corresponding to self-loops on the modes, and the remaining entries either 0 or 1 depending on the particular CV cluster state. Starting from an $N$-mode square cluster state defined by this nullifier and carrying out the measurement pattern described for the ideal cluster, we generate the finitely squeezed Kitaev lattice with complex nullifiers $\hat{a}_{s}^{\prime}=\hat{a}_{s}=\left(\hat{p}_{s, 1}+\hat{p}_{s, 2}+\right.$ $\left.\hat{p}_{s, 3}+\hat{p}_{s, 4}\right)=0$ and $\hat{b}_{f}^{\prime}=\hat{b}_{f}-i e^{-2 r_{1}} \hat{p}_{f, 1}+i e^{-2 r_{2}} \hat{p}_{f, 2}-$ $i e^{-2 r_{3}} \hat{p}_{f, 3}+i e^{-2 r_{4}} \hat{p}_{f, 4}=0$, for the remaining modes, where $s$ and $f$ label the stars and plaquettes, respectively, and $\partial f$ denotes the boundary of a face. Comparing with the nullifiers in the infinitely squeezed limit, we observe that finite squeezing introduces extra imaginary terms to the plaquette nullifiers. The stabilizers corresponding to these complex nullifiers are $A_{s}^{\prime}(\xi)=$ $e^{-i \xi \hat{a}_{s}^{\prime}}=\prod_{j \in \operatorname{star}(s)} X_{s, j}(\xi)$ and $B_{f}^{\prime}(\eta)=e^{-i \eta \hat{b}_{f}^{\prime}}=$ $\prod_{j \in \partial(f)} Z_{f, j}\left((-1)^{j} \eta\right) \exp \left[\eta(-1)^{j} e^{-2 r_{j}}\left(\eta+\hat{p}_{j}\right)\right]$. These operators correspond to the Kitaev model with an extra complex term, but note that these reduce exactly to the Kitaev stabilizers as the squeezing parameter $r \rightarrow \infty$. These new stabilizers still commute and so the new state $|\phi\rangle$ corresponds to the anyonic ground state with $A_{s}^{\prime}(\xi)|\phi\rangle=|\phi\rangle$ and $B_{f}^{\prime}(\eta)|\phi\rangle=|\phi\rangle$. In order to see the effects of finite squeezing, we apply the unphysical stabilizers to the physical ground state,

$$
\begin{aligned}
& B_{f}(\eta)|\phi\rangle=\exp \left[-i \eta \hat{b}_{f}\right]|\phi\rangle {\left[\eta \sum_{j=1}^{4}(-1)^{j+1} e^{-2 r_{j}}\left(\hat{p}_{j}+\eta\right)\right] } \\
&=\exp {\left[-\eta \sum_{j=1}^{4}(-1)^{j+1} e^{-2 r_{j}}\left(\hat{p}_{j}+\eta\right)\right] \exp \left[-i \eta \hat{b}_{f}\right]|\phi\rangle } \\
& \times \exp {\left[-\eta \sum_{j=1}^{4}(-1)^{j+1} e^{-2 r_{j}}\left(\hat{p}_{j}+\eta\right)\right] B_{f}^{\prime}(\eta)|\phi\rangle } \\
&=\exp {\left[-\eta \sum_{j=1}^{4}(-1)^{j+1} e^{-2 r_{j}}\left(\hat{p}_{j}+\eta\right)\right]|\phi\rangle } \\
&=\exp
\end{aligned}
$$

where we dropped the subscripts $f$ of the momentum operators for simplicity. We observe that finite squeezing of the ground state violates the unphysical stabilizer conditions by an imaginary phase, $\sim i \eta^{2}$, and by imaginary position shifts, $\sim i \eta$. Having derived the form of our finitely squeezed $\mathrm{CV}$ lattice, we now turn to anyonic excitations and basic braiding operations. 


\section{ANYONIC CREATION AND BRAIDING ON FINITELY SQUEEZED LATTICE}

By applying single-mode operations to the ground state, we can examine the excitations above the physical ground state. The effects of finite squeezing on the creation of anyonic excitations are revealed when we calculate the violation of the finitely squeezed nullifiers due to the application of $X_{i}(t)$ and $Z_{i}(t)$ on some mode of the physical ground state defined by

$$
\begin{aligned}
& A_{s}^{\prime}(\xi)|\phi\rangle=e^{-i \xi \hat{a}_{s}^{\prime}}|\phi\rangle=|\phi\rangle, \\
& B_{f}^{\prime}(\eta)|\phi\rangle=e^{-i \eta \hat{b}_{f}^{\prime}}|\phi\rangle=|\phi\rangle .
\end{aligned}
$$

Note that now, due to the non-Hermiticity of the physical nullifiers, their violations through anyonic excitations may in general be complex. An excitation of the vertex ground state due to $Z_{j}(t)$ corresponds to

$$
A_{s}^{\prime}(\xi)\left[Z_{j}(t)|\phi\rangle\right]=e^{-i \xi \hat{a}_{s}^{\prime}} e^{i t \hat{x}_{j}}|\phi\rangle
$$

but $\hat{a}_{s}^{\prime}=\hat{a}_{s}$, as the finite squeezing has no effect on the vertex nullifier. Hence,

$$
\begin{aligned}
A_{s}^{\prime}(\xi)\left[Z_{j}(t)|\phi\rangle\right] & =e^{-i \xi \hat{a}_{s}} e^{i t \hat{x}_{j}}|\phi\rangle \\
& =e^{\xi t\left[\hat{a}_{s}, \hat{x}_{j}\right]} Z_{j}(t) A_{s}(\xi)|\phi\rangle \\
& =e^{-i \xi t} Z_{j}(t) A_{s}^{\prime}(\xi)|\phi\rangle \\
& =e^{-i \xi t}\left[Z_{j}(t)|\phi\rangle\right], \forall \xi \in \mathbb{R} .
\end{aligned}
$$

Just as in the infinitely squeezed case, this yields a stabilizer violation of $t$. The $Z_{j}(t)$ applied to the plaquette stabilizers gives

$$
\begin{aligned}
B_{f}^{\prime}(\eta)\left[Z_{j}(t)|\phi\rangle\right] & =e^{-i \eta \hat{b}_{f}^{\prime}} e^{i t \hat{x}_{j}}|\phi\rangle \\
& =e^{\eta t\left[\hat{b}_{f}^{\prime}, \hat{x}_{j}\right]} Z_{j}(t) B_{f}^{\prime}(\eta)|\phi\rangle \\
& =e^{\eta t\left[-i e^{-2 r_{j}} \hat{p}_{f, j}, \hat{x}_{j}\right]}\left[Z_{j}(t)|\phi\rangle\right] \\
& =e^{-\eta t e^{-2 r_{j}}}\left[Z_{j}(t)|\phi\rangle\right], \forall \eta \in \mathbb{R} .
\end{aligned}
$$

This differs from the infinitely squeezed case (where we had no violation at all), with an imaginary nullifier violation of $i t e^{-2 r_{j}}$. This time physical anyons may appear as complex violations of the ground-state stabilizers. Applying $X_{j}(t)$ to the ground state yields,

$$
\begin{aligned}
A_{s}^{\prime}(\xi)\left[X_{j}(t)|\phi\rangle\right] & =e^{-i \xi \hat{a}_{s}^{\prime}} e^{-i t \hat{p}_{j}}|\phi\rangle \\
& =e^{-i \xi \hat{a}_{s}} e^{-i t \hat{p}_{j}}|\phi\rangle \\
& =X_{j}(t) A_{s}^{\prime}(\xi)|\phi\rangle \\
& =\left[X_{j}(t)|\phi\rangle\right], \forall \xi \in \mathbb{R},
\end{aligned}
$$

so no stabilizer violation occurs. Finally, the plaquette stabilizer gives

$$
\begin{aligned}
B_{f}^{\prime}(\eta)\left[X_{j}(t)|\phi\rangle\right] & =e^{-i \eta \hat{b}_{f}^{\prime}} e^{-i t \hat{p}_{j}}|\phi\rangle \\
& =e^{-\eta t\left[\hat{b}_{f}^{\prime}, \hat{p}_{j}\right]} X_{j}(t) B_{f}^{\prime}(\eta)|\phi\rangle \\
& =e^{-\eta t\left[\hat{b}_{f}, \hat{p}_{j}\right]} X_{j}(t) B_{f}^{\prime}(\eta)|\phi\rangle \\
& =e^{ \pm i \eta t}\left[X_{j}(t)|\phi\rangle\right], \forall \eta \in \mathbb{R},
\end{aligned}
$$

which corresponds to a violation of $\pm t$.

In summary, finite squeezing gives us a violation of the plaquette ground state stabilizer due to the action of $Z(t)$, whereas it did not exhibit violations in the infinitely squeezed case. The vertex stabilizers are unaffected and yield violations of the same form as in the infinitely squeezed case. This suggests that any topological operations carried out on $e$ anyons are topologically protected from error. However, the only operation possible on star anyons alone is the SUM gate which is not on its own sufficient for quantum computation. The anyons are now represented in general by complex violations. These may then said to be new types of excitations produced through finite squeezing which we call complex anyons.

The effects on the braiding procedure and hence gate operations are determined by generating vertex and plaquette anyons and guiding them around each other in closed loops.

$$
\begin{aligned}
\left|\phi_{f i n}\right\rangle & =\left[Z_{1}(-t) Z_{2}(t) Z_{3}(-t) Z_{4}(t)\right]\left|\phi_{i n i}\right\rangle \\
& =\left[Z_{1}(-t) Z_{2}(t) Z_{3}(-t) Z_{4}(t)\right] X_{k}(s)|\phi\rangle .
\end{aligned}
$$

Commuting through to enact the braid yields

$$
\begin{gathered}
\left|\phi_{\text {fin }}\right\rangle=\exp [i s t] X_{k}(s)\left[Z_{1}(-t) Z_{2}(t) Z_{3}(-t) Z_{4}(t)\right]|\phi\rangle \\
=\exp [i s t] X_{k}(s) \exp \left[-t \sum_{j=1}^{4}(-1)^{j+1} e^{-2 r_{j}}\left(\hat{p}_{j}+t\right)\right] B_{f}^{\prime}(t)|\phi\rangle .
\end{gathered}
$$

Using our definition of the ground state, $B_{f}^{\prime}(t)|\phi\rangle=|\phi\rangle$, we obtain

$$
\begin{aligned}
\left|\phi_{\text {fin }}\right\rangle & =\exp [i s t] X_{k}(s) \exp \left[-t \sum_{j}(-1)^{j+1} e^{-2 r_{j}}\left(\hat{p}_{j}+t\right)\right]|\phi\rangle \\
& =\exp [i s t] \exp \left[-t \sum_{j}(-1)^{j+1} e^{-2 r_{j}}\left(\hat{p}_{j}+t\right)\right]\left|\phi_{i n i}\right\rangle .
\end{aligned}
$$

We can express the term proportional to $t$ as an imaginary displacement,

$$
\begin{aligned}
\left|\phi_{f i n}\right\rangle= & \exp [i s t] \exp \left[-t^{2} \sum_{j}(-1)^{j+1} e^{-2 r_{j}}\right] \\
& \times \prod_{j} X_{j}\left(-i t(-1)^{j+1} e^{-2 r_{j}}\right)\left|\phi_{i n i}\right\rangle .
\end{aligned}
$$

As in the infinitely squeezed case, we observe a phase change of $e^{i s t}$, but this time, for finite squeezing, we have an extra imaginary displacement and a term proportional to $t^{2}$ (corresponding to an extra imaginary phase, similar to what we had before for the groundstate plaquette stabilizers with finite squeezing). We 
may call the combination of these terms the topological factor for the braiding of finitely squeezed anyons, and we note that this factor would not be obtained if the initial states were unexcited. We can absorb the complex displacement into the definition of the ground state such that the nullifer $\hat{b}_{f}$ is no longer zero, but has an imaginary violation $\hat{b}_{f}=i t \sum_{j}(-1)^{j+1} e^{-2 r_{j}}$. Then $\hat{b}_{f}=s+i t \sum_{j}(-1)^{j+1} e^{-2 r_{j}}=s^{\prime}$ is the nullifier corresponding to a finitely squeezed $m$-anyon. The topological phase produced when braided with an $e$-type anyon is then

$$
\exp \left[i s^{\prime} t\right]=\exp \left[i\left(s+i t \sum_{j}(-1)^{j+1} e^{-2 r_{j}}\right) t\right]
$$

This is one of the central results of this paper, extending the simple (infinite squeezing) factor $e^{i s t}$ of [16] to the realistic case of finite squeezing. Similar to the infinitely squeezed case, the state can acquire any phase, but now the phase is modified by extra factors due to finite squeezing.

This should not affect topological gate operations since these displacements and dampings are taken into account through the definition of the ground state and they depend on the known squeezing $r_{j}$ available at each qumode. Hence we have shown that topological operations on CV abelian anyons are protected from errors due to finite squeezing of the initial ground state. Note that this result goes beyond that of Ref. [16]. There it was argued that in the case of finite squeezing, excited (anyonic) states can be experimentally distinguished from the ground state and, similarly, the effects of braiding loops can still be detected, provided the corresponding phase-space displacements are sufficiently large. In our treatment, such a requirement is unnecessary. Using the complex-nullifier formalism, we find that any finitesqueezing effects on the 1st-moment-shifts can be absorbed into the definition of the excited states as well as into the topological phases. However, note that these re-defined WH frames would always depend on the explicit values of the corresponding anyonic excitations and anyon-braidings [i.e., the $t$-dependencies in Eqs. (21-30)].

As a result, all those gates shown to be implementable in a topological fashion turn out to be robust against finite squeezing errors. Nonetheless, non-topological operations such as the Fourier transform do pick up extra errors due to finite squeezing since they rely on the ability to create zero-momentum eigenstates. In fact, all these non-topological gates include 2nd-or higher-moment manipulations which will be affected by the finite squeezing of the initial graph states. Similarly, of course, the entanglement of the ground and excited states, becoming manifest through nonclassical 2nd-moment correlations, does depend on the finite squeezing of the initial states.

\section{CONCLUSION}

We have shown that abelian anyons generated on an ideal CV Kitaev lattice are a useful resource for continuous-variable quantum computation. We have described the quantum gates that can be achieved by topological operations alone and found this did not form a sufficiently powerful gate set. By including offline squeezing, measurements, and cubic phase gates, we have shown that the computational power can be increased significantly. These additional resources provide a universal gate set for the $\mathrm{CV}$ anyons. It has therefore been possible for us to give a classification of the topological operations available to $\mathrm{CV}$ abelian anyons. We have shown that braiding and fusion only account for controlled and uncontrolled WH gates, non-topological operations are essential to complete the Clifford group, and a further non-Gaussian element is required to achieve universality. We note that the topological controlled displacements only give entanglement (including 2nd-moment non-classical correlations) if we have access to superpositions of anyonic excitations, e.g. in a bi-anyonic vacuum state, $\int d s d t e^{-s^{2}-t^{2}}|s\rangle|t\rangle$. Such an extension will give rise to a more general model including non-abelian anyons.

We have also considered the effect of finite squeezing on the CV Kitaev lattice and found that the excitations on this physical ground state correspond to Gaussian anyon states. These physical anyons, mathematically represented by complex stabilizer violations, can perform fault-tolerant topological operations that are protected against errors due to finite squeezing. However, we cannot create a fully protected gate set, since some gates require non-topological operations. Nonetheless, we have been able to identify those operations which are protected, and in a future work, we shall attempt to present a universal, topological gate set using nonabelian anyons.

This research has been supported by the EU STREP project COMPAS FP7- ICT-2007-C-212008 under the FET-Open Programme, by the Scottish Universities Physics Alliance (SUPA) and by the Engineering and Physical Sciences Research Council (EPSRC). DFM acknowledges the financial support from the Deutscher Akademischer Austausch Dienst (DAAD). PvL acknowledges support from the Emmy Noether program of the DFG.
[1] Cedric Beny and Ognyan Oreshkov, Phys. Rev. Lett. 104, 120501 (2010)
[2] E. Novais and Harold U. Baranger, Phys. Rev. Lett. 97, 040501 (2006) 
[3] M. A. Nielsen and I.L. Chuang. Quantum Computation and Information. Cambridge University Press, second edition, 2000.

[4] P. W. Shor, Phys. Rev. A 52, R2493 (1995)

[5] F. Wilczek, Phys. Rev. Lett 48, 1144 (1982)

[6] J. Preskill, Lecture notes on topological quantum computation, http://www.theory.cltech.edu /people/preskill/ph219/topological.ps

[7] X. G. Wen, Quantum Field Theory of Many-Body Systems, Oxford University, Oxford, (2004)

[8] A. Kitaev, Ann Phys. 303, 2 (2003)

[9] L. Hormozi, G. Zikos, N. E. Bonesteel and S. H. Simon, Phys. Rev. B 75, 165310 (2007)

[10] Chetan Nayak, Steven H. Simon, Ady Stern, Michael Freedman, and Sankar Das Sarma, Rev. Mod. Phys. 80, 1083 (2008)

[11] J. R. Wootton and J. K. Pachos, arXiv:0904.4373v2[quant-ph], (2009)

[12] Liang Jiang, Charles L. Kane, and John Preskill, Phys. Rev. Lett. 106, 130504 (2011)

[13] Parsa Bonderson and Roman M. Lutchyn, Phys. Rev. Lett. 106, 130505 (2011)
[14] A. Kitaev, Ann Phys. 321, 2 (2006)

[15] Y. J. Han, R. Raussendorf, and L. M. Duan, Phys. Rev. Lett., 98, 150404 (2007)

[16] Jing Zhang, Changde Xie, Kunchi Peng, and Peter van Loock, Phys. Rev. A 78, 052121 (2008)

[17] S. D. Bartlett et al., Phys. Rev. Lett. 88, 097904 (2002).

[18] Robert Raussendorf, Daniel E. Browne, and Hans J. Briegel, Phys. Rev. A 68, 022312 (2003)

[19] J. Zhang and S. L. Braunstein, Phys. Rev. A 73, 032318 (2006)

[20] N. C. Menicucci et al., Phys. Rev. Lett. 97, 110501 (2006)

[21] P. van Loock, C. Weedbrook, and M. Gu, Phys. Rev A 76, 032321 (2007)

[22] N. C. Menicucci, S. T. Flammia, and P. van Loock, Phys. Rev. A 83, 042335 (2011)

[23] D. Gottesman, A. Kitaev, and J. Preskill, Phys. Rev. A 64, 012310 (2001)

[24] Note that in standard CV encoding, the $C_{x}$ and $C_{z}$ gates are symplectic operations involving squeezers and beam splitters. In the abelian-anyon encoding, however, they would only result in controlled shifts. 